\title{
The Paradigm Shift in Telecommunications Services and Networks
}

\author{
Masayoshi Ejiri \\ Service and Network Operations Section, Network Engineering Department \\ NTT Service Engineering Headquarters \\ 1-6 Uchisaiwaicho 1-Chome Chiyoda-ku, Tokyo 100-19 Japan \\ Telephone: +81-3-3509-5320 \\ Fax: +81-3-5251-7801 \\ E-mail: ejiri@nw.hqs.cae.ntt.jp
}

\begin{abstract}
Liberalization of the telecommunications market has fundamentally changed the situation faced by every player involved with providing telecommunications services and networks. This paper provides an overview of the ongoing evolution of telecommunications and outlines the paradigm shift that will be necessary in such areas as service provision, network architecture and pricing.

\section{Keywords}

Customer contact, customer-defined service, customer premises equipment, information agent function, information provider, negotiations, network architecture, operation system function, service attributes, service provider, service providing structure, virtual service provider
\end{abstract}

\section{INTRODUCTION}

Changes in the telecommunications market are being driven by rapidly advancing technology and customer demand for increasingly sophisticated services. Successfully coping with the situation will require a paradigm shift in the way providers look at how they provide services. Currently, telecommunications services are offered through complicated interworked networks by various service providers using an array of rapidly evolving technologies. A new mechanism is needed to 
ensure that service providers can offer their services in a manner that looks and feels seamless from the perspective of the customer.

Customers may also want to freely choose services and then negotiate the terms of those services. To meet this demand, a negotiating mechanism for use between customers and service providers is essential. In the multimedia area, broadband services will require new pricing policies that enable providers to maximize the use of network resources and to offer lower prices to end customers.

Another essential feature of multimedia services is easy and economical access to specific information. Faced with a tremendous volume of available information, customers will want to edit this information to make it better serve their needs. An agent function for information providers becomes a key to meeting this customer demand.

To meet the needs of the new telecommunications business environment, new concepts in service, operations, management and networks must be established. These concepts cannot be built upon existing ways of thinking about telecommunications service. Instead, they require a fundamental paradigm shift in thinking toward a full realization that the multimedia era has arrived. This paper discusses the causes of the paradigm shift, the nature of the new paradigm and the crucial issues which must be dealt with under the altered circumstances.

\section{REVOLUTION IN THE SERVICE PROVISION STRUCTURE}

The liberalization of the telecommunications market and the resulting keen competition among providers has created a complex service environment where many service providers offer services through mutually interworked networks. With Plain Old Telephone Service (POTS), services are provided via a simple mechanism, shown in Figure 1. In this service provision structure, a carrier constructs the network and plays the role of service provider as well. Carriers deploy standardized network architecture and interfaces based on CCITT (currently ITU-T) standards. Network elements are produced in compliance with these standards. As a result, POTS service provides seamless service to customers on a global basis.

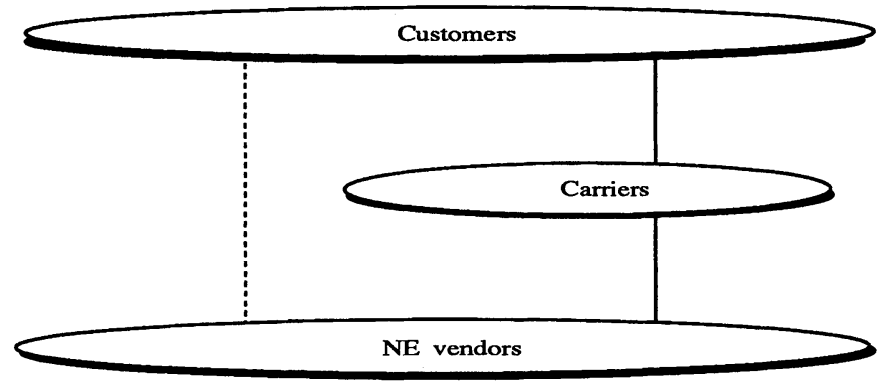

Figure 1 Traditional telephone service provision structure. 
Recent advances in computer technology have brought significant improvements in Customer Premises Equipment (CPE). Furthermore, many service providers (SPs) now specialize in the full utilization of CPE capabilities with services such as Value-Added Network (VAN). The result is that several service providers now work in combination to meet customer needs (see Figure 2). Under this service provision structure, SPs and vendors tend to deploy their own network architecture and interfaces in an effort to make them de facto standards. This complicates the service provision structure and reduces customer choice, since customers may avoid otherwise desirable services to prevent an interface mismatch (Ejiri, 1994a, 1994b).

To avoid inconvenience to customers, customers, SPs and vendors need to cooperate in order to create a seamless web out of their diverse services (Network Management Forum, 1994). A virtual service provider - which integrates several service providers - could be a solution. The activities of standardizing bodies (ITU-T, ISO, etc.) and various consortia (ATM Forum, Network Management Forum, etc.) are also expected to help resolve these issues.

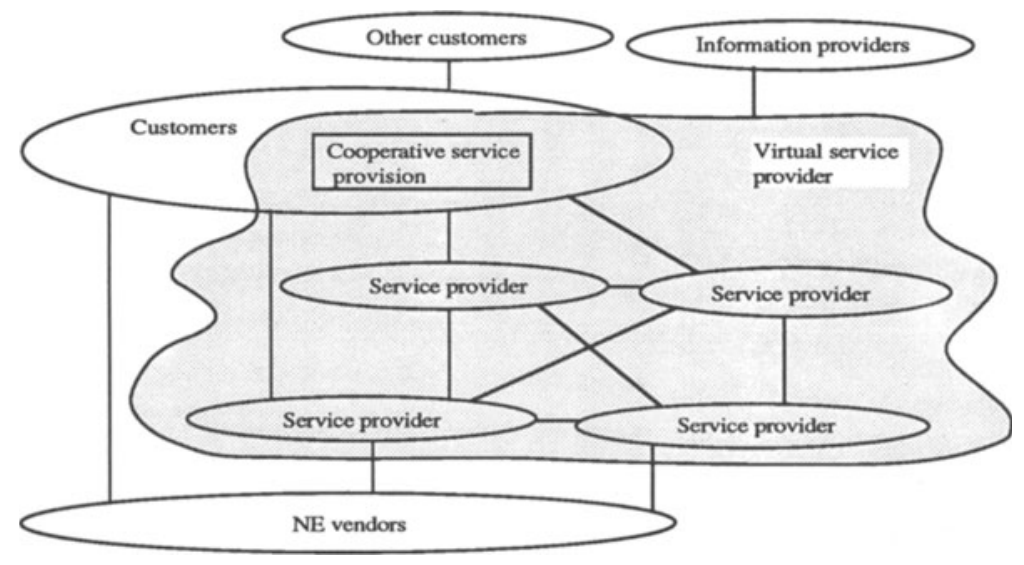

Figure 2 Future service provision structure.

\section{SERVICE OFFERINGS BASED UPON CUSTOMER NEGOTIATIONS}

The rapid diversification of customer demand means some customers feel restricted in their choice of SPs and vendors. Customers may also want to exert more control over service attributes and conditions. The most important issue is pricing, which is closely related to service attributes such as traffic characteristics and QOS (Quality of Service).

Due to the price of SP telecommunications, various measures have been implemented within CPE networks to minimize computer communications costs. If SPs offer lower prices along with 
improved CPE-SP linkage, total costs will be reduced. This will greatly stimulate the market for broadband/high-speed communications. Therefore, in computer-based multimedia networks, new concepts and functions for communication conditions negotiations are essential. Moreover, these must be consistent with the network utilization strategy between SPs and customers.

Though lower prices are urgently needed, especially for long distance broadband video communications, efforts to reduce network cost and improve bandwidth compression technology may not be enough to achieve the target price. The situation is more promising for VBR (Variable Bit Rate) video communications and high-speed computer communications. The burst-intensive nature of traffic for these services will permit network utilization strategies (based on quality variation and available time selection) that enable lower prices.

Figure 3 shows a diagram of the basic service provision structure with customer negotiations (Ejiri, 1994a). Negotiations may have static (pre-assigned) and dynamic (on demand) features. Service attributes subject to negotiation include time (point and delay), QOS, addressing and pricing. Customers negotiate with several SPs on these attributes and select their own service conditions and prices based on what's offered. SPs negotiate with customers to use their network resources at a level near $100 \%$, obtaining maximum benefit with minimum investment.

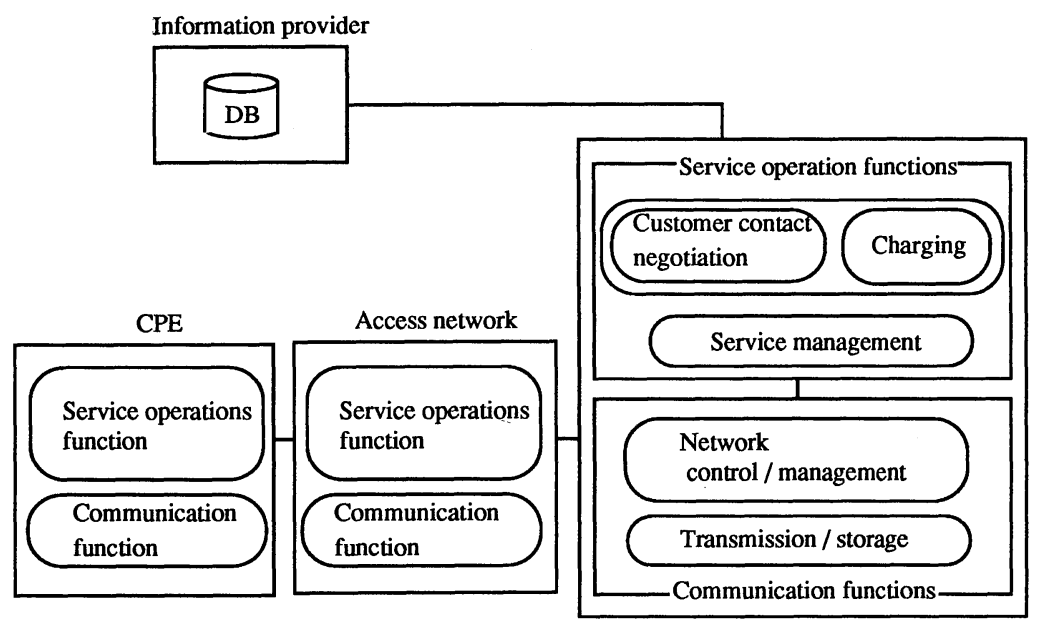

Figure 3 Basic service provision structure including customer negotiation.

In SP networks the functions indicated in Figure 4 need to be subject to negotiation. The service negotiation function manages the negotiation process based on customer demand and network resources/service status. The network resource management function allocates network resources to individual customers following requests from the service negotiation function. Various database systems tracking real-time status information on services and networks must be constructed and 
maintained to support the negotiation process. To ensure efficient negotiations, negotiation scenarios and intelligent functions involving the connection between CPE and SP networks will have to be developed.

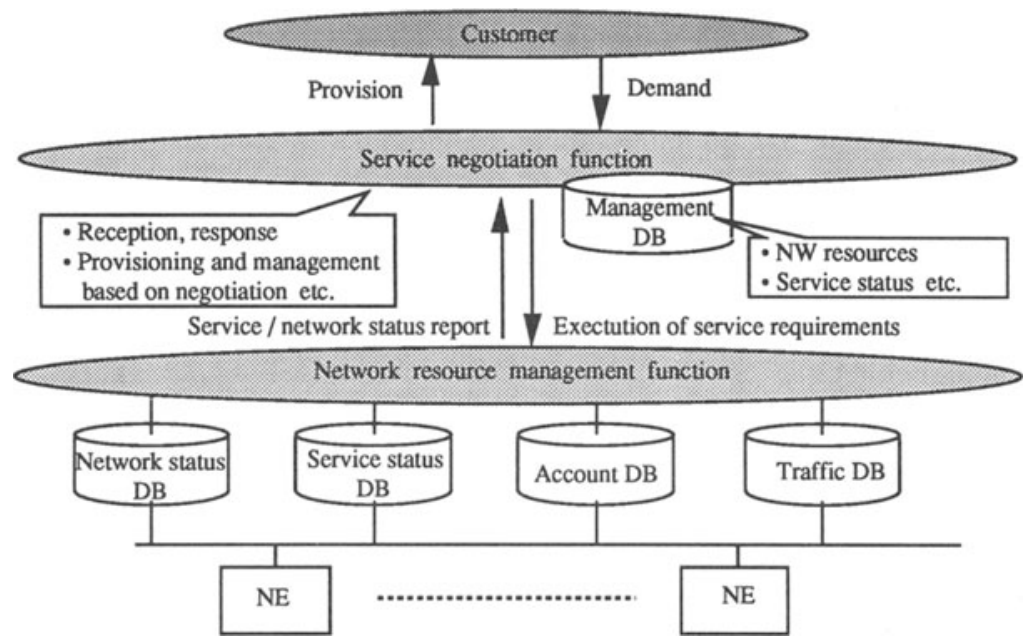

Figure 4 Function allocation for negotiations.

\section{EVOLUTION OF CUSTOMER-DEFINED SERVICES}

The above discussion about the negotiation of service attributes and conditions concerns only a primitive first step. Customers will want more than the ability to select services from a menu offered by SPs. They will want to change service parameters themselves, combining aspects of various services in a manner that best meets their individual needs. The trend toward customized service is currently being studied as UPT (universal personal telecommunications) by the ITU-T (ITU-T, 1994b).

Network resources will have to be rapidly and flexibly interconnected using sophisticated network operations and management functions. In order to establish the proper architecture, a layering concept should be introduced in order to identify the various functional components. This approach is similar to that used for computer networks.

In the new network architecture, both CPE and SP networks can be based on the same concept and make use of the same software packages (see Section 7). With both CPE and SP networks based on the same concept, many possibilities arise for communication among layered functions (see Figure 5). Customers and SPs can then utilize each others' functional capabilities. The 
reference points identified in each layer in Figure 5 are important for identifying new interfaces based on network functional architecture.

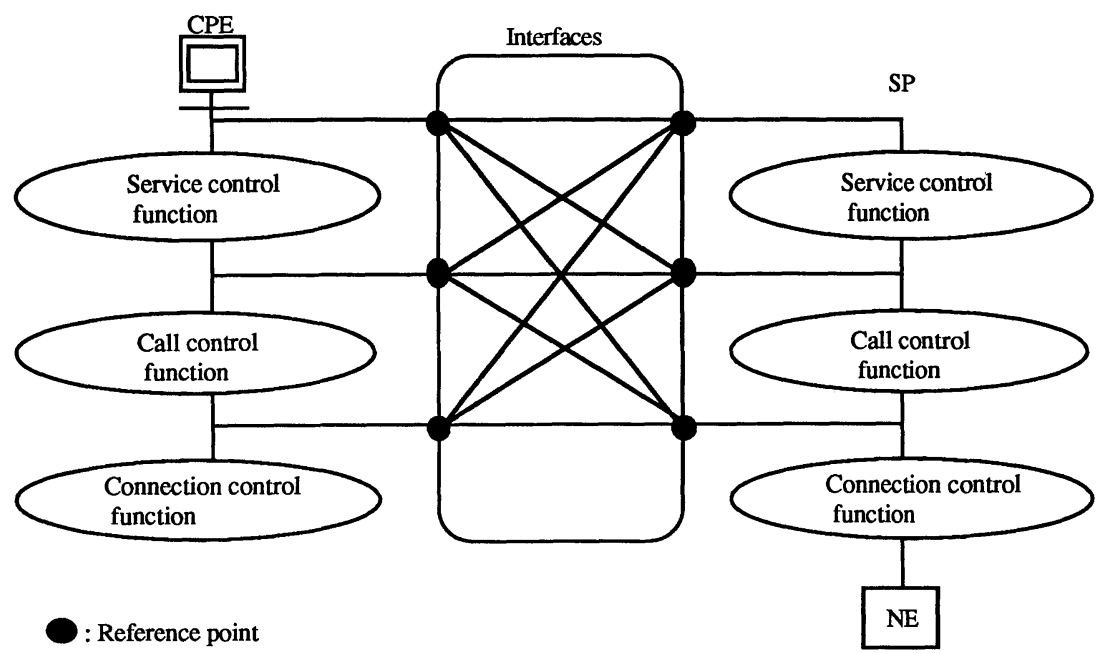

Figure 5 CPE-SP interface examples.

\section{ENRICHMENT OF INFORMATION PROVIDER SERVICES}

Information providers (IPs) and content providers are expected to play major roles in popularizing multimedia communications services. Since IPs use SP networks to distribute information to customers, cooperation between IPs and SPs is very important.

Attractive, useful information for customers is usually expensive to produce. A stable mechanism for drawing back costs and generating profit is essential for the success of multimedia communication services. At the same time, the mechanism should ensure that customers have easy access to the information, as well as a way to compare various features of information (quality, price, acquisition procedure, etc.) from different IPs.

Sometimes, customers have only fragmentary data about what they need and it is important that they still have a way to access the needed information, if possible. For example, a customer may know what information they need but not know which IP is best for such information. Conversely, they may know a good IP but have only the barest notion about the information they need. To satisfy various types of customer requests, an information agent function will be needed (Natarajan and Slawsky, 1992). Figure 6 shows a diagram of such a function. In this example, some possible functions are added, such as accounting for information charges and electronic 
mailing using an IP database as a mailbox. Using this function, customers can smoothly obtain the desired information and also add new information producing new multimedia information to be sent on. This capability will accelerate the acceptance of multimedia communications.

IPs will usually collect charges from individual customers who access the information. If SPs and IPs cooperate, another charging procedure could be implemented. Information could be free of charge to individual customers, leading to greatly increased traffic and greater income for the SP which could then be shared with the IP. This service strategy could well benefit all concerned. Customers would receive free information, while the IP and SP might both enjoy greater profits.

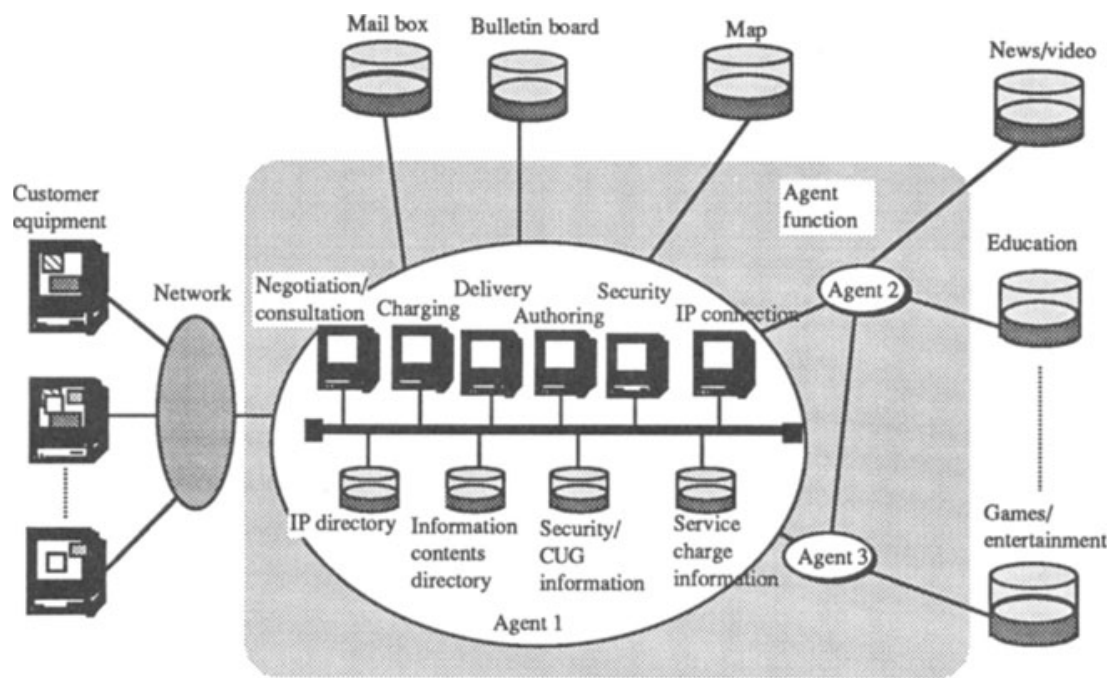

Figure 6 Agent functionality schematic.

\section{IMPROVED CUSTOMER CONTACT}

Negotiations between the SP and customer over service conditions will be carried out primarily through a machine-machine interface (MMI). To realize rapid, accurate and economical negotiations, the negotiation interface will have to be largely mechanized. The interface for customer contact will gradually move away from contact with humans to contact with machines (see Figure 7). The operations system function (OSF) diagrammed in Figure 7 includes negotiation and operation functions for reconfiguration, testing and related tasks. 


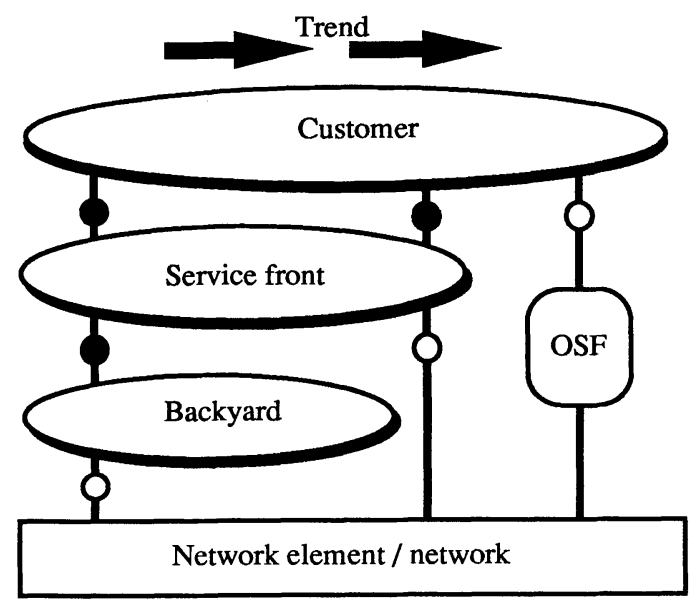

Human to Human
Interface
Machine to
Machine Interface

OSF: Operation service function (with security check function)

Figure 7 Operation service interfaces.

Although the mechanization of the customer contact function releases operators from their jobs on the SP side and thereby reduces operating costs, it is important to avoid the imposition of complicated procedures upon customers. To increase customer convenience, the deployment of intelligent functions in both CPE and SP networks will be necessary.

In a sophisticated, mechanized telecommunications environment, customers will want to know more about operations and management functions, as well as service capabilities (ITU-T, 1994a). In order to satisfy this demand for information, it is necessary to retain a powerful Human to Human Interface (HHI) which removes as much inconvenience as possible in communications. To provide support to operators at the HHI service front, SPs have developed sophisticated management information networking capabilities. Operators are able to obtain appropriate information quickly and efficiently when contacted by customers (ITU-T, 1993).

OSF and the customer contact point (service front) are equally important in the future service environment. HHI and MMI complement each other, offering customers the best type of contact for the information needed by customers at a particular moment.

\section{NEW PARADIGM FOR NETWORK ARCHITECTURE}

Many new service providers have entered into the telecommunications market, leading to a complex and rapidly changing telecommunications service provision structure. Since customers want their telecommunications services to work in a seamless fashion, it is necessary to deploy a new network architecture capable of achieving this. This, in turn, necessitates a paradigm shift in the way service providing mechanisms are conceptualized. 
A diagram of the existing network is shown in Figure 8. CPE and SP networks are developed independently and are interconnected through User Network Interface (UNI). Within SP networks, the transport network and various network nodes are interconnected through Network Node Interface (NNI). ITU-T SG15 currently discusses UNI and Service Node Interface (SNI) in access networks (Matsushita, Okazaki and Yoshida, 1995).

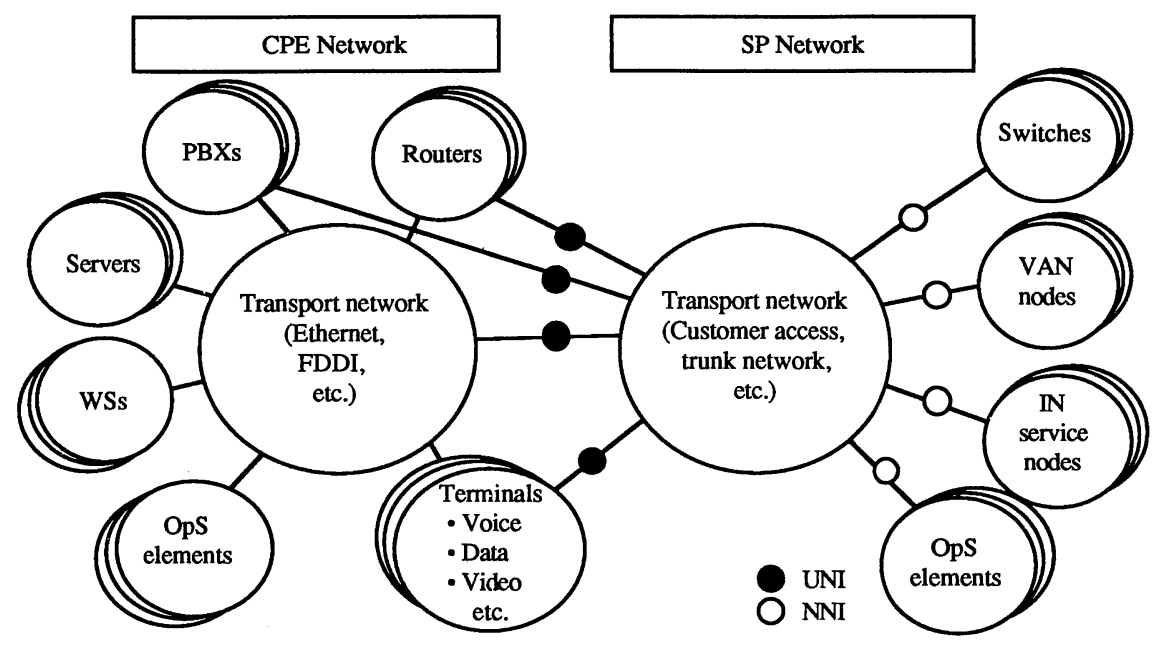

Figure 8 Existing network architecture and interfaces.

As technology advanced, the difference between CPE and SP networks narrowed. Within the CPE environment, the common architecture included a centralized information processing network based on a mainframe computer. The progress of LAN (Local Area Network) and WAN (Wide Area Network) technologies introduced distributed processing architecture into the local and world-wide environments.

In SP networks, network nodes (switching systems) used to be based on a mainframe type functionally centralized architecture, although they were distributed geographically. The need for rapid and flexible service provisioning as well as sophisticated services has forced a review of existing network architecture. The answer is a functionally distributed architecture, such as separation of service definition functions and connection functions.

Digitalization of transmission is proceeding in both CPE and SP networks. CPE networks have evolved using digital transmission technology as is the case, for example, with the Ethernet. With SP networks, digital transmission systems have been introduced into trunk networks as a first step. The digitalization process for CPE and SP networks has proceeded independently. Once access networks in SP networks are digitalized, a fence will be removed between CPE and SP networks. 
CPE and SP networks are now evolving in a similar direction - toward a functionally distributed environment using similar digital processing and transmission technologies. In the emerging environment, it will be possible to use the same hardware and software for the two types of network.

Suppose the trend continues, the networks merge into functional homogeneity and one unique class of interface is established which is supported by the use of common software packages. The new network architecture shown in Figure 9 would be shared between customers and SPs. This would accelerate the smooth interworking of CPE and SP networks in the complex service offering environment illustrated in Figure 2. Customers would have the freedom to construct their networks without feeling constrained with respect to the choice of SPs or vendors when choosing services. Some customers could become SPs for other customers, thus expanding on their business opportunities.

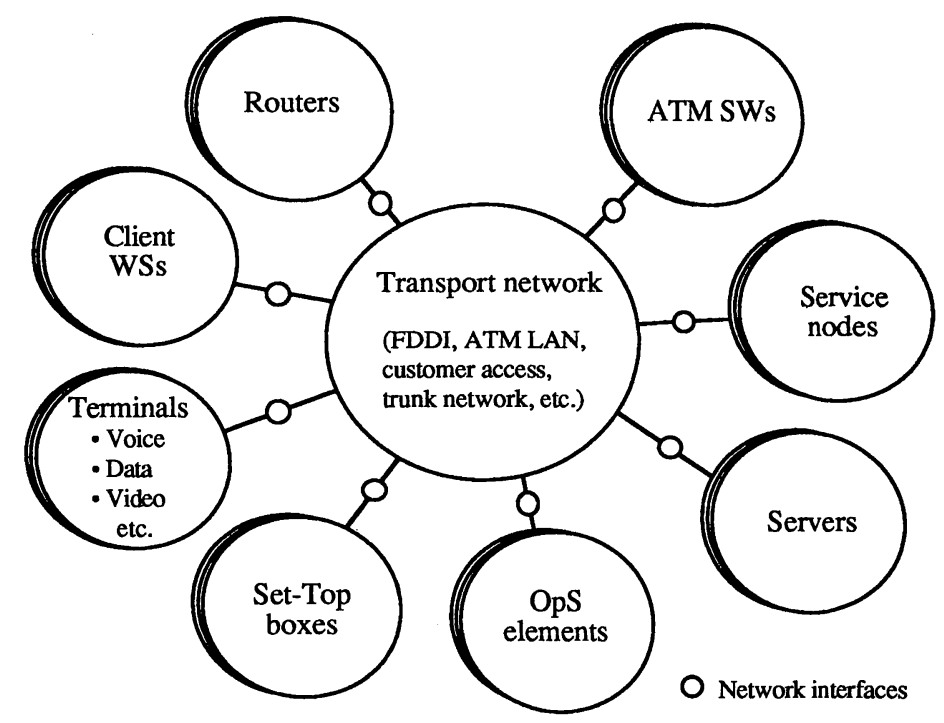

Figure 9 New network architecture and interfaces.

\section{CONCLUSION}

The service provision structure is becoming complex, involving a number of overlapping fields, as well as several service providers within most of these fields. Customers still want seamless 
service, even though they want to freely choose services from any combination of SPs and negotiate over price and service features.

To satisfy these customer demands, service providers will need to undergo a paradigm shift in the way they think about service provision. The new paradigm, from a technological standpoint, involves an agent/negotiation function as well as new network architecture integrating the service providers' networks with CPE networks in a distributed processing environment. This paradigm shift also involves a shift in pricing structure to accommodate the widespread advent of multimedia communications.

\section{ACKNOWLEDGEMENTS}

The author would like to express his gratitude to Messrs. Masahiko Matsushita and Noriyuki Terada for their pertinent suggestions during the preparation of this paper.

\section{REFERENCES}

Ejiri, M. (1994a) For whom the advancing service/network management. Keynote speech, NOMS ‘94 Symposium Record, Vol. 2, pp. 422-433.

Ejiri, M. (1994b) Advancing service operations and operations systems. NTT Review, Vol. 6, No. 3, pp. 31-36, 1994.

ITU-T (1993) Tele administration service. ITU-T Draft Recommendation F.ADM.

ITU-T (1994a) Framework recommendation on functional access networks. ITU-T Draft Recommendation G.9xx

ITU-T (1994b) Universal Personal Telecommunications (UPT) service description. ITU-T Recommendation F.851.

Maeda, M. and M. Ejiri (1994) Enhancement of service front operation, NTT Review, Vol. 6, No. 3, pp. 37-45.

Matsushita, M., T. Okazaki and M. Yoshida (1995) A telecommunications management integration network IEICE TRANS. COMMUN., Vol. E78-B, No. 1 (January).

Natarajan, N. and G. M. Slawsky (1992) A framework architecture for multimedia information networks. IEEE Communications Magazine, February, pp. 97-104.

Network Management Forum (1994) Requirement capture: service management automation and re-engineering (draft), September 23. 


\section{BIOGRAPHY}

Masayoshi Ejiri received his Bachelor's degree in Engineering from the University of Tokyo in 1967. Since joining NTT, he has worked in a number of areas, including transmission systems development and network planning and engineering. He has also directed a telephone office and managed operations systems development and telecommunications software production. He is currently in charge of strategy and system development for the Service and Network Operations Section of NTT's Network Engineering Department. Mr. Ejiri is a member of IEEE and is the General Co-Chair of IEEE/IFIP 1996 Network Operations and Management Symposium (NOMS '96). 